\title{
FURTHER STUDY ON ONE-DIMENSIONAL SHOCK WAVES IN NONLINEAR ELASTIC MEDIA*
}

\author{
BY \\ T. C. T. TING \\ University of Illinois at Chicago Circle
}

\begin{abstract}
One may define the growth of a shock wave by the growth in the amplitude of discontinuity in the velocity (denoted by $[v]$ ) across the shock wave as the shock wave propagates. One may also define the growth of a shock wave by the growth in the amplitude of discontinuity in the stress [ $\sigma]$, strain $[\epsilon]$, or entropy $[\eta]$. It is shown that one definition predicts the growth of the shock wave while others may predict its decay. In this paper we derive the transport equations for one-dimensional shock waves in nonlinear elastic media in which the shock wave can be defined as the amplitude of either $[\nu],[\sigma],[\epsilon]$ or $[\eta]$. Moreover, the dependent quantity can be any one of, or a linear combination of, the seven quantities behind the shock wave. It is shown that when the region ahead of the shock wave is under a homogeneous deformation, the amplitudes of $[v],[\sigma]$ and $[\epsilon]$ grow or decay simultaneously if $(a)[\epsilon]^{2}$ is a strictly increasing function of $[\eta]$, or $(b)$ the purely mechanical theory of shock waves is employed in which the effect of the entropy is ignored. Regardless of whether the effect of the entropy is ignored or not, there is no assurance that the amplitudes of $[v],[\sigma]$ and $[\epsilon]$ grow or decay simultaneously if the region ahead of the shock wave is not under a homogeneous deformation.
\end{abstract}

1. Introduction. It is well-known in the theory of acceleration waves that the growth of the discontinuity in the acceleration across a singular surface is invariably accompanied by the growth of the discontinuities in other quantities such as the strain rate and stress gradient. In the theory of shock waves, the growth of the discontinuity in, say, the velocity across a shock wave is not necessarily accompanied by the growth in other discontinuities such as the stress, strain or entropy. Therefore, whether a shock wave grows or decays depends on whether one looks at the growth or decay of the discontinuity in the velocity, stress, strain or entropy. Moreover, the growth or decay of a shock wave depends, among others, upon a certain quantity behind the shock wave. This quantity can be the strain gradient [1, 2], but can also be the acceleration [3], the stress gradient, the strain rate, etc., or a linear combination of these quantities. If we define the "critical jump in the strain gradient" as the one which holds the key to the growth or decay of the discontinuity in the strain across the shock wave, then one has to define a different "critical jump in strain gradient" which holds the key to the growth or decay of the discontinuity in, say, the stress across the shock wave.

\footnotetext{
* Received December 6, 1978; revised version received April 26, 1979. The work presented here is supported by the U.S. Army Research Office-Durham under Grant DAAG 29-76-G-0121 through the University of Illinois at Chicago Circle. The author is grateful to Dr. T. W. Wright for valuable comments which led to the consideration of the limiting cases $\xi=0$ and $\xi=1$ in the paper.
} 
The purpose of this paper is twofold. First, the relations between all relevant quantities across the shock wave in one-dimensional nonlinear elastic media are derived in a concise form by using matrix notations. Secondly, the growth of the amplitudes of the discontinuities in the velocity, stress, strain and entropy as well as the quantities behind the shock wave (such as the acceleration, strain gradient, etc.) are all related to a single parameter, namely, the acceleration of the shock wave. With this parameterization, a transport equation can be obtained in which one is free to choose any discontinuity as the definition of shock wave amplitude and any quantity behind the shock wave as the one which holds the key to the growth or decay of the shock wave. Moreover, it is readily seen from this parameterization whether the discontinuities in the velocity, stress and strain grow and decay simultaneously.

2. Basic equations. For waves propagating in a one-dimensional homogeneous medium, let $\sigma, \epsilon, v$ and $\rho$ be the stress, strain, velocity and mass density, respectively. The equation of motion and the continuity condition can be written as

$$
\begin{gathered}
\sigma_{x}+\rho b=\rho v_{t}, \\
v_{x}=\epsilon_{t},
\end{gathered}
$$

where $b$ is the body force which is assumed to be continuous, $x$ is the reference coordinate, $t$ is the time and the subscript implies partial differentiation. We will assume that the stress $\sigma$ and the absolute temperature $\theta$ can be derived from the internal energy $e$ :

$$
e=e(\epsilon, \eta), \quad \sigma=\partial e / \partial \epsilon, \quad \theta=\partial e / \partial \eta,
$$

where $\eta$ is the entropy. Since $\sigma=\sigma(\epsilon, \eta)$,

$$
\begin{gathered}
\sigma_{t}=E \epsilon_{t}+G \eta_{t}, \\
E=\partial \sigma / \partial \epsilon, \quad G=\partial \sigma / \partial \eta .
\end{gathered}
$$

We will also assume that heat conduction is negligible. Hence

$$
\eta_{t}=0 .
$$

Eqs. (1), (2), (4) and (6), which completely define the wave propagation in one-dimensional nonlinear elastic media, can be written concisely as

$$
A \mathbf{w}_{t}+\mathbf{B} \mathbf{w}_{x}=\mathbf{b}
$$

where

$$
\begin{array}{rlrl}
\mathbf{A} & =\left[\begin{array}{cccc}
\rho & 0 & 0 & 0 \\
0 & 0 & 1 & 0 \\
0 & 1 & -E & -G \\
0 & 0 & 0 & 1
\end{array}\right], & \mathbf{w}=\left[\begin{array}{c}
v \\
\sigma \\
\epsilon \\
\eta
\end{array}\right], \\
\mathbf{B}=\left[\begin{array}{cccc}
0 & -1 & 0 & 0 \\
-1 & 0 & 0 & 0 \\
0 & 0 & 0 & 0 \\
0 & 0 & 0 & 0
\end{array}\right], & \mathbf{b}=\left[\begin{array}{c}
-\rho b \\
0 \\
0 \\
0
\end{array}\right]
\end{array}
$$


3. Equations governing the shock waves. Across a shock wave where discontinuities in $\sigma, \epsilon, v$ and $\eta$ occur, we will define the discontinuity in a quantity $f$ by

$$
[f]=f^{-}-f^{+}
$$

where $f^{-1}$ and $f^{+}$are the values of $f$ immediately behind and just in front of the shock wave. Eqs. (1) and (2) are then replaced by

$$
\begin{gathered}
{[\sigma]=-\rho U[v],} \\
{[v]=-U[\epsilon]}
\end{gathered}
$$

where $U$ is the speed of the shock wave. Notice that the mass density $\rho$ is referred to the reference configuration and hence is a constant. We define the total differentation of a quantity $f$ along the shock wave by

$$
d f / d t=f_{x} U+f_{t} .
$$

Applying Eq. (12) to Eqs. (10) and (11), we obtain

$$
\begin{gathered}
d[\sigma] / d t+\rho U(d[v] / d t)=-\rho[v](d U / d t), \\
d[v] / d t+U(d[\epsilon] / d t)=-[\epsilon](d U / d t) .
\end{gathered}
$$

Total differentiation of $\sigma=\sigma(\epsilon, \eta)$ and use of Eq. (5) yields

$$
\frac{d[\sigma]}{d t}=E^{-} \frac{d[\epsilon]}{d t}+G^{-} \frac{d[\eta]}{d t}+[E] \frac{d \epsilon^{+}}{d t}+[G] \frac{d \eta^{+}}{d t},
$$

where the following relation has been used:

$$
[f g]=f^{-}[g]+[f] g^{+} .
$$

Finally, the balance of energy requires that [4]

$$
-U\left[e+\frac{1}{2} \rho v^{2}\right]=[\sigma v] .
$$

This can be rewritten in several alternate forms. One of the forms is (see the Appendix)

$$
[e]-\frac{1}{2} \rho U^{2}[\epsilon]^{2}=\sigma^{+}[\epsilon] .
$$

Since

$$
\frac{d[e]}{d t}=\left[\sigma \frac{d \epsilon}{d t}\right]+\left[\theta \frac{d \eta}{d t}\right]=\sigma^{-} \frac{d[\epsilon]}{d t}+[\sigma] \frac{d \epsilon^{+}}{d t}+\theta^{-} \frac{d[\eta]}{d t}+[\theta] \frac{d \eta^{+}}{d t},
$$

by using Eq. (16), the total differentiation of Eq. (18) yields, after using Eqs. (19) and (10),

$$
\theta^{-} \frac{d[\eta]}{d t}=\rho U[\epsilon]^{2} \frac{d U}{d t}+[\epsilon] \frac{d \sigma^{+}}{d t}-[\sigma] \frac{d \epsilon^{+}}{d t}-[\theta] \frac{d \eta^{+}}{d t} .
$$

Eqs. (13), (14), (15) and (20) can be put in the following concise form:

$$
\left(U \mathbf{A}^{-}-\mathbf{B}\right) \frac{d[\mathbf{w}]}{d t}=\mathbf{g} \frac{d U}{d t}+\mathbf{h}
$$

where A, B and $w$ are defined in Eq. (8) and 


$$
\begin{aligned}
& \mathbf{g}=\left[\begin{array}{c}
-\rho[v] \\
-[\epsilon] \\
0 \\
\rho U^{2}[\epsilon]^{2} / \theta
\end{array}\right], \\
& \mathbf{h}=\left[\begin{array}{c}
U\left([E] \frac{d \epsilon^{+}}{d t}+[G] \frac{d \eta^{+}}{d t}\right) \\
0 \\
\frac{U}{\theta^{-}}\left([\epsilon] \frac{d \sigma^{+}}{d t}-[\sigma] \frac{d \epsilon^{+}}{d t}-[\theta] \frac{d \eta^{+}}{d t}\right)
\end{array}\right]
\end{aligned}
$$

Notice that $\mathbf{h}=\mathbf{0}$ if the region ahead of the shock wave is under a homogeneous deformation. We assume that, at a certain time $t,[\mathbf{w}]$ and $U$ are known at the shock wave and proceed to find the relations for the variation of these quantities as the shock wave propagates.

4. Transport equations for shock waves. We will now use Eqs. (7) and (21) to derive the transport equations for the growth or decay of the shock wave. To begin with, we rewrite Eq. (21) as

$$
\frac{d[\mathbf{w}]}{d t}=\alpha \frac{d U}{d t}+\boldsymbol{\beta}
$$

where

$$
\begin{gathered}
\alpha=\mathbf{T g}, \quad \beta=\mathbf{T h}, \\
\mathbf{T}=\left(U \mathbf{A}^{-}-\mathbf{B}\right)^{-1} .
\end{gathered}
$$

Next, since $\mathbf{B}$ and $\mathbf{b}$ are continuous, Eqs. (7) and (16) yield

$$
\mathbf{A}^{-}\left[\mathbf{w}_{t}\right]+\mathbf{B}\left[\mathbf{w}_{x}\right]=-[\mathbf{A}] \mathbf{w}_{t}^{+}
$$

If we eliminate $\left[\mathbf{w}_{x}\right]$ between Eqs. (26) and the relation

$$
\frac{d[\mathbf{w}]}{d t}=U\left[\mathbf{w}_{x}\right]+\left[\mathbf{w}_{t}\right]
$$

we have

$$
\left(U \mathbf{A}^{-}-\mathbf{B}\right)\left[\mathbf{w}_{\imath}\right]=-\left(\mathbf{B} \frac{d[\mathbf{w}]}{d t}+U[\mathbf{A}] \mathbf{w}_{t}^{+}\right) .
$$

Hence, by Eqs. (23) and (25),

$$
\begin{gathered}
{\left[\mathbf{w}_{t}\right]=-\phi(d U / d t)-\psi,} \\
\phi=\mathbf{T} \mathbf{B} \boldsymbol{\alpha}, \quad \psi=\mathbf{T} \mathbf{B} \boldsymbol{\beta}+U \mathbf{T}[\mathbf{A}] \mathbf{w}_{t}^{+} .
\end{gathered}
$$

By substituting Eqs. (23) and (29) into (27), we obtain

$$
\left[\mathbf{w}_{x}\right]=\frac{1}{U}(\alpha+\phi) \frac{d U}{d t}+\frac{1}{U}(\beta+\psi) .
$$


Eq. (23) is the transport equation for [w] with $d U / d t$ as the parameter. $d U / d t$ can be related to any component of $\left[w_{t}\right]$ or $\left[w_{x}\right]$ through Eqs. (29) and (31). Notice that, with the exception of $d U / d t$, the right-hand sides of Eqs. (23), (29) and (31) are known. Also notice that $\mathbf{T}$, the inverse of $\left(U \mathbf{A}^{-}-\mathbf{B}\right)$ as defined in Eq. (25), can be shown to be

$$
\mathbf{T}=\frac{1}{\left(E^{-}-\rho U^{2}\right)}\left[\begin{array}{cccc}
-U & E^{-} & 1 & G^{-} \\
E^{-} & -\rho U E^{-} & -\rho U & -\rho U G^{-} \\
1 & -\rho U & -1 / U & -G^{-} / U \\
0 & 0 & 0 & \left(E^{-}-\rho U^{2}\right) / U
\end{array}\right]
$$

We assume that $E^{-} \neq \rho U^{2}$. The case $E^{-}=\rho U^{2}$ will be discussed later. We now write Eqs. (23), (31) and (29) explicitly in the following.

$$
\begin{aligned}
\frac{d[v]}{d t} & =-\frac{(1+\xi) \tau-\xi}{(1-\xi) \tau}[\epsilon] \frac{d U}{d t}+\frac{U Q}{1-\xi}, \\
\frac{d[\sigma]}{d t} & =\frac{\rho U(2 \tau-\xi)}{(1-\xi) \tau}[\epsilon] \frac{d U}{d t}-\frac{\rho U^{2} Q}{1-\xi}, \\
\frac{d[\epsilon]}{d t} & =\frac{(2 \tau-1) \xi}{U(1-\xi) \tau}[\epsilon] \frac{d U}{d t}-\frac{Q}{1-\xi}, \\
\frac{d[\eta]}{d t} & =\frac{\rho U}{\theta^{-}}[\epsilon]^{2} \frac{d U}{d t}+\frac{1}{\theta^{-}}\left\{[\epsilon] \frac{d \sigma^{+}}{d t}-[\sigma] \frac{d \epsilon^{+}}{d t}-[\theta] \frac{d \eta^{+}}{d t}\right\} ; \\
{\left[v_{x}\right] } & =\frac{\xi\{(\xi+3) \tau-2 \xi\}}{U(1-\xi)^{2} \tau}[\epsilon] \frac{d U}{d t}-\left\{\frac{2 \xi Q}{(1-\xi)^{2}}+\frac{q}{1-\xi}\right\}, \\
{\left[\sigma_{x}\right] } & =-\frac{\rho\{(3 \xi+1) \tau-\xi(1+\xi)\}}{(1-\xi)^{2} \tau}[\epsilon] \frac{d U}{d t}+\rho U\left\{\frac{(1+\xi) Q}{(1-\xi)^{2}}+\frac{q}{1-\xi}\right\}, \\
{\left[\epsilon_{x}\right] } & =-\frac{\xi\{(3 \xi+1) \tau-(3 \xi-1)\}}{U^{2}(1-\xi)^{2} \tau}[\epsilon] \frac{d U}{d t}+\frac{1}{U}\left\{\frac{(3 \xi-1) Q}{(1-\xi)^{2}}+\frac{q}{1-\xi}\right\}, \\
{\left[\eta_{x}\right] } & =\frac{1}{U} \frac{d[\eta]}{d t} ; \\
{\left[v_{t}\right] } & =\frac{1}{\rho}\left[\sigma_{x}\right], \\
{\left[\sigma_{t}\right] } & =\frac{\rho U\{(\xi+3) \tau-2 \xi\}}{(1-\xi)^{2} \tau}[\epsilon] \frac{d U}{d t}-\rho U^{2}\left\{\frac{2 Q}{(1-\xi)^{2}}+\frac{q}{1-\xi}\right\}, \\
{\left[\epsilon_{t}\right] } & =\left[v_{x}\right], \quad\left[\eta_{t}\right]=0,
\end{aligned}
$$

where

$$
\begin{gathered}
\xi=\rho U^{2} / E^{-}, \quad \tau=\theta^{-} /\left(G^{-}[\epsilon]\right), \quad q=[E] \epsilon_{t}^{+} / E^{-}, \\
Q=\frac{1}{E^{-} \tau}\left\{\left(E^{+}+\tau[E]-\frac{[\sigma]}{[\epsilon]}\right) \frac{d \epsilon^{+}}{d t}+\left(G^{+}+\tau[G]-\frac{[\theta]}{[\epsilon]}\right) \frac{d \eta^{+}}{d t}\right\} .
\end{gathered}
$$

It follows from Eqs. (36) that $Q=q=0$ when (i) the region ahead of the shock wave is under a homogeneous deformation, or (ii) the purely mechanical theory is used in which $\sigma$ is a 
function of $\epsilon$ only and $E^{-}=E^{+}=\rho U^{2}$. Case (ii) includes the special case of linear elastic materials in which $E$ is a constant.

Eqs. (33)-(35) hold when $U \neq 0,0<\xi<1$ and the shock wave is stable. However, Eqs. (33)-(35) may be evaluated for the limiting values of $\xi=0$ and $\xi=1$. (See Section 6.)

When we eliminate $d U / d t$ between one of Eqs. (33) and an equation obtained by a linear combination of equations in (34), (35), we obtain a transport equation in the form

$$
d[f] / d t=\gamma(\lambda-[g])
$$

where $f$ can be either $v, \sigma, \epsilon$ or $\eta$ and $g$ can be either one of, or a linear combination of, $v_{x}$, $\sigma_{x}, \epsilon_{x}, \eta_{x}, v_{t}, \sigma_{t}$ and $\epsilon_{t} \cdot \gamma$ and $\lambda$ are known quantities and will have different expressions depending on the choice of $f$ and $g$. Regardless of the choice of $f$ and $g, \lambda$ vanishes when $Q$ and $q$ vanish. We list below three examples of Eq. (37) in which $f=v, \sigma$ and $\epsilon$, respectively, while $g=\epsilon_{x}$ for all three examples.

$$
\begin{aligned}
f=v, & g=\epsilon_{x} \\
\gamma=-\frac{U^{2}(1-\xi)\{(1+\xi) \tau-\xi\}}{\xi\{(3 \xi+1) \tau-(3 \xi-1)\}}, & \lambda=\frac{1}{U(1-\xi)}\left\{q-\frac{\tau Q}{(1+\xi) \tau-\xi}\right\}, \\
\gamma=\frac{U E^{-}(1-\xi)(2 \tau-\xi)}{(3 \xi+1) \tau-(3 \xi-1)}, & \quad \lambda=\frac{1}{U(1-\xi)}\left\{q+\frac{(2-3 \xi) \tau Q}{2 \tau-\xi}\right\}, \\
f=\epsilon, & g=\epsilon_{x} \cdot \\
\gamma=\frac{U(1-\xi)(2 \tau-1)}{(3 \xi+1) \tau-(3 \xi-1)}, & \lambda=\frac{1}{U(1-\xi)}\left\{q-\frac{3 \tau Q}{(2 \tau-1)}\right\} .
\end{aligned}
$$

Eq. (38c) can be shown to be identical to the results obtained in [1] for $\lambda=0$ and in [2] for $\lambda \neq 0$. It is seen that $\gamma$ and $\lambda$ assume different forms even though we choose the same $g$. In Eqs. (38) $\lambda$ can be regarded as the "critical jump in strain gradient" because $d[f] / d t=0$ when $\left[\epsilon_{x}\right]=\lambda$ (see [2]). We see that the "critical jumps in strain gradient" are not the same for $d[v] / d t, d[\sigma] / d t$ and $d[\epsilon] / d t$.

5. Discussion. From Eqs. (10) and (11), $[\sigma]$ and $[\epsilon]$ always have the same sign while [v] has the opposite sign. In comparing the growth or decay of these amplitudes, we should compare $-d[v] / d t, d[\sigma] / d t$ and $d[\epsilon] / d t$. If they have the same sign, then the amplitudes of $[v],[\sigma]$ and $[\epsilon]$ either grow or decay simultaneously. Because of different expressions for $\gamma$ and $\lambda$ as shown in Eqs. $(38 \mathrm{a}, \mathrm{b}, \mathrm{c})$, we conclude that $-d[\nu] / d t, d[\sigma] / d t$ and $d[\epsilon] / d t$ may not have the same sign.

When the region ahead of the shock wave is under a homogeneous deformation and hence $Q=0$, it follows from Eqs. (33) that $-d[v] / d t, d[\sigma] / d t$ and $d[\epsilon] / d t$ will have the same sign if

$$
\tau>1 / 2 \quad \text { or } \quad \tau<\xi / 2
$$

and will have different signs if

$$
\xi / 2<\tau<1 / 2
$$


If one assumes that $[\epsilon]^{2}$ is a strictly increasing function of $[\eta]$, then from Eqs. $(33 c, d)$, with $Q=q=0$,

$$
\frac{d[\epsilon]^{2}}{d[\eta]}=\frac{2 \theta^{-}(2-1 / \tau)}{E^{-}(1-\xi)}>0
$$

Noticing that $0<\xi<1$, the inequality is satisfied if

$$
\tau>1 / 2 \text { or } \tau<0 \text {. }
$$

Since Eq. (42) satisfies the inequalities in Eq. (39), we conclude that for shock waves propagating into a region of homogeneous deformation, $-d[v] / d t, d[\sigma] / d t$ and $d[\epsilon] / d t$ always have the same sign if $[\epsilon]^{2}$ is a strictly increasing function of $[\eta]$.

Finally, for the purely mechanical theory of shock waves in which the entropy is ignored, Eqs. (33-35) and (38) are simplified by letting

$$
\tau=\infty, \quad Q=\left([E] / E^{-}\right)\left(d \epsilon^{+} / d t\right) .
$$

In particular, Eqs. (33) can be written as

$$
\begin{aligned}
-\frac{d[v]}{d t} & =\frac{1+\xi}{1-\xi}[\epsilon] \frac{d U}{d t}-\frac{U[E]}{E^{-}(1-\xi)} \frac{d \epsilon^{+}}{d t}, \\
\frac{1}{\rho U} \frac{d[\sigma]}{d t} & =\frac{2}{1-\xi}[\epsilon] \frac{d U}{d t}-\frac{U[E]}{E^{-}(1-\xi)} \frac{d \epsilon^{+}}{d t}, \\
U \frac{d[\epsilon]}{d t} & =\frac{2 \xi}{1-\xi}[\epsilon] \frac{d U}{d t}-\frac{U[E]}{E^{-}(1-\xi)} \frac{d \epsilon^{+}}{d t} .
\end{aligned}
$$

One way to see whether $-d[v] / d t, d[\sigma] / d t$ and $d[\epsilon] / d t$ have the same sign or not is to see if they vanish for the same value of $d U / d t$. From Eq. (44) it is clear that they do not. Thus in the neighborhood of, say, $d[v] / d t=0$, they will have different signs. However, in the particular case when the region ahead of the shock wave is under a homogeneous deformation and hence $d \epsilon^{+} / d t=0$, Eq. (44) shows that $-d[v] / d t, d[\sigma] / d t$ and $d[\epsilon] / d t$ always have the same sign. Thus, for shock waves propagating into a region of homogeneous deformation, $-d[v] / d t, d[\sigma] / d t$ and $d[\epsilon] / d t$ always have the same sign if the purely mechanical theory of shock waves is employed.

6. The limiting cases $\xi=0$ and $\xi=1$. For $\xi=0$, if we assume that $U \neq 0$ and $\sigma, \epsilon, v$ and $G^{-}$remain bounded, we have $E^{-}=\infty, q=\epsilon_{t}^{+}, Q=(d \epsilon / d t)^{+}, v_{x}{ }^{-}=\epsilon_{t}^{-}=0$ and $\epsilon_{x}{ }^{-}=0$. Eq. (33c), (34a) and (34c) then reduce to trivial identities while the rest of Eqs. (33-35) are simplified by letting $\xi=0$.

For $\xi=1$, the matrix $T$ defined in Eq. (32) does not exist. However, by expanding each term of Eqs. (33-35) into a power series of $(1-\xi)$ and comparing the coefficients of equal power in $(1-\xi)$, we obtain as $\xi \rightarrow 1$, assuming that $[\epsilon] \neq 0$ and $\tau \neq 1 / 2$,

$$
\begin{array}{rlrl}
d U / d t & =U Q /(2 \tau-1)[\epsilon], & \frac{d[v]}{d t}=-\frac{U}{2}\left(q-\frac{\tau Q}{2 \tau-1}\right), \\
\frac{d[\sigma]}{d t}=\frac{\rho U^{2}}{2}\left(q+\frac{\tau Q}{2 \tau-1}\right), & \frac{d[\epsilon]}{d t}=\frac{1}{2}\left(q-\frac{3 \tau Q}{2 \tau-1}\right) .
\end{array}
$$

We see that the growth or decay of the shock wave no longer depends on the quantity be- 
hind the shock wave when $\xi=1$. In particular, for linear materials $Q=q=0$ and Eqs. (45) reduce to the well-known results that $U,[v],[\sigma]$ and $[\epsilon]$ are constants as the shock wave propagates.

7. Concluding remarks. The results obtained here can be extended to materials other than nonlinear elastic media. However, one need not drive the transport equations explicitly to be convinced that the amplitudes of the discontinuities in the velocity, stress and strain may not grow or decay simultaneously. To see this, we eliminate $d U / d t$ between Eqs. (13) and (14) and make use of Eq. (11) to obtain

$$
\frac{d[\sigma]}{d t}+\rho U^{2} \frac{d[\epsilon]}{d t}=-2 \rho U \frac{d[v]}{d t}
$$

Therefore, unless $-d[v] / d t, d[\sigma] / d t$ and $d[\epsilon] / d t$ always vanish at the same instant, they will have different signs at least in the neighborhood of the time at which one of them vanishes. It is important to point out that Eq. (46) is independent of any constitutive assumptions. Consequently, the fact that the amplitudes of $[v],[\sigma]$ and $[\epsilon]$ may not grow or decay simultaneously applies to materials other than nonlinear elastic media also.

It seems possible to apply the approach used here in deriving the transport equations for one-dimensional shock waves to three-dimensional shock waves. This has been done for the special case of three-dimensional nonlinear elastic fluids. The results are strikingly similar to the results obtained here.

\section{REFERENCES}

[1] P. J. Chen and M. E. Gurtin, The growth of one-dimensional shock waves in elastic non-conductors, Int. J. Solids Structures 7, 5-10 (1971)

[2] P. J. Chen, One-dimensional shock waves in elastic non-conductors, Arch. Rat. Mech. Anal. 43, 350-362 (1971)

[3] T. W. Wright, An intrinsic description of unsteady shock waves, Q. J. Appl. Math. Mech. 29, 311-324 (1976)

[4] C. A. Truesdell and R. A. Toupin, The classical field theories, Handbuch der Physik III/1, Springer, 225-293 (1960)

Appendix: balance of energy across a shock wave. From Eqs. (10) and (11), we have the well-known relation for the shock wave speed $U$,

$$
[\sigma]=\rho U^{2}[\epsilon] .
$$

By using the relation

$$
[f g]=[f][g]+f^{+}[g]+g^{+}[f]
$$

we expand Eq. (17) as

$$
-U[e]-1 / 2 \rho U\left\{[v]^{2}+2 v^{+}[v]\right\}=[\sigma][v]+\sigma^{+}[v]+v^{+}[\sigma] .
$$

If we replace $[\sigma]$ and $[v]$ in terms of $[\epsilon]$ by using Eqs. (11) and (A1), we obtain

$$
[e]-1 / 2 \rho U^{2}[\epsilon]^{2}=\sigma^{+}[\epsilon]
$$

which is Eq. (18). If the region ahead of the shock wave is stress-free, $\sigma^{+}=0$ and we have

$$
[e]=1 / 2 \rho U^{2}[\epsilon]^{2}=1 / 2[\sigma][\epsilon]
$$

where the last equality is from Eq. (A1). 


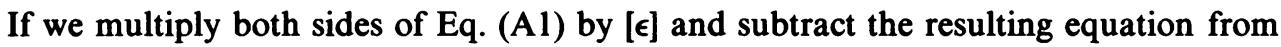
Eq. (A4), we have

$$
[e]+1 / 2 \rho U^{2}[\epsilon]^{2}=\sigma^{-}[\epsilon] .
$$

This is derived in [1]. Addition of Eqs. (A4) and (A6) yields

$$
[e]=\left(\sigma^{+}+\sigma^{-}\right)[\epsilon] / 2 \text {. }
$$

The right-hand side of this equation is the area under the secant between $\sigma\left(\epsilon^{+}, \eta^{+}\right)$and $\sigma\left(\epsilon^{-}, \eta^{-}\right)$on the $\sigma \sim \epsilon$ curve. 